\title{
17.
}

\section{Die lineale Erzeugung von Curven dritter Ordnung.}

(Von dem Herrn Professor Dr. Gra/'smann, Oberlehrer am Gymnasio zu Steltin.)

Im 31. Bande (S. 123 ff.) und im 36. Bande dieses Journals (S. $178 \mathrm{ff}$.) habe ich drei möglichst einfache Methoden angegeben, um sämmtliche Curven dritter Ordnung durch Bewegung gerader Linien um feste oder geradlinige bewegliche Puncte zu erzeugen. Es wurden diese Methoden durch die drei Gleichungen :

$$
\begin{array}{r}
x a B c D x D_{1} c_{1} B_{1} a_{1} x=0, \\
x a A a_{1} \cdot x b B b_{1} \cdot x c=0, \\
x a A \cdot x b B \cdot x c C=0,
\end{array}
$$

dargestellt, in welchen die kleinen Buchstaben Puncte, die grofsen gerade Linien, $\boldsymbol{x}$ den die Curve construirenden Punct bezeichnen, und in welchen die Multiplication planimetrisch ist. Ich habe dort streng und ausführlich nachgewiesen, dafs die beiden ersten Methoden allgemein sind, d.h. dafs sich jede Curve dritter Ordnung durch jede dieser Methoden erzeugen lasse; und zwar die erste auch dann, wenn man $\boldsymbol{B}$ und $\boldsymbol{B}_{1}$ zusammenfallen läfst, wogegen ich für die dritte Methode dort den Beweis nur mehr andeutete als ausführte (Bd. 36. S. 180).

Hr. Prof. Bellavitis behauptet nun in einem Aufsatze (unter dem Titel: Sopra un algoritmo proposto per esprimere gli allineamenti etc. Venezia 1855), welchen er mir zuzuschicken die Güte gehabt hat, es sei keine jener Methoden allgemein, sondern es liefsen sich durch jede derselben nur ganz specielle Curven dritter Ordnung erzeugen; nämlich nur solche, die durch 7 oder gar durch 6 Puncte bestimmt werden, und die daher von noch speciellerer Nalur seien, als die durch 8 Puncte bestimmte Doppelpunctscurve; dagegen sei Chasles der erste, welcher (Comple rendu, 30 mai 1853) ein solches Problem gelöset habe. Dies, und die freie Darstellung des Algorithmus, wie ich ihn in Band 31, 36, 42, 44 dieses Journals entwickelt, so wie der Rechnungsregeln, welche ich dort für diesen Algoritlımus mitgetheilt habe, bildet den Inhalt des genannten Aufsalzes. Der Einwurf mufste für mich um so bedeutender sein, da $\mathrm{Hr}$. Bellaritis diesen Algorithmus selbständig behandelt 
und damit Resultate erzielt. Ich glaube es daher mir und dem Gegenstande schuldig zu sein, denselben noch einmal aufzunehmen, und namentlich auch die Allgemeinheit der dritten Methode streng nachzuweisen. Zugleich werde ich zeigen, dafs sich nicht nur jede beliebige lineale Erzeugung der Curven dritter Ordnung auf die zweite Methode direct zurückführen läfst, sondern dafs sich auch aus 9 beliebig gegebenen Puncten die Constanten der Gleichung (2.) so bestimmen lassen, dafs die durch diese Gleichung dargestellte Curve durch jene 9 Puncte geht; $d$. h. ich werde die linealen Eigenschaften eines Zelinecks, welches einer Curve dritter Ordnung eingeschrieben ist, aus einer Gleichung von jener Form ableiten. Gelegentlich werde ich dann auch die Fehlschlüsse angeben, auf welchen der Einwurf des Hrn. Bellavitis beruht.

\$. 1 .

Die wichtigsten Rechnungsregeln der planimetrischen Multiplication.

Im folgenden, wie in den früheren Aufsätzen, sollen stets die Puncle mit kleinen, die geraden Linien mit gro/sen Buchstaben bezeichnet werden, und jene "Elemente erster Stufe," diese "Elemente zweiter Stufe" heifsen, während ich die Zahlen „Grö/sen nullter Stufe" nennen werde. Ich werde vorzugsweise solche Gleichungen betrachten, deren eine Seite Null, und deren andere Seite ein Product ist, in welchem die Summe der Stufenzahlen aller darin enthaltener Factoren durch drei theilbar ist, und in welchem überhaupt keine andere Verknüpfung vorkommt, als nur planimetrische Multiplication. Eine solche Gleichung drückt, wenn nicht einer der Factoren Null ist, stets aus, dafs ein bestimmter Punct in einer bestimmten geraden Linie liegt. So z. B. drückt die Gleichung

$$
\boldsymbol{A} \boldsymbol{b}=\mathbf{0}
$$

aus, dafs der Punct $\boldsymbol{b}$ in der geraden Linie $\boldsymbol{A}$ liegt. Hr. Bellavitis braucht hiefür das Wort Congruenz; was aber nicht ganz angemessen sein dürfte, da ein Punct nicht mit einer geraden Linie congruent genannt werden kann. Ich werde mich, wo es auf einen kurzen Namen ankommt, des entsprechenden Wortes Incidenz bedienen, so dafs also die obige Gleichung, welche ausdrückt, dafs $b$ in $\boldsymbol{A}$ fällt, oder $\boldsymbol{b}$ mit $\boldsymbol{A}$ incident ist, eine Incidenz heifsen soll, während ich für das Zusammenfallen sweier Puncte oder sweier gerader Linien das Wort Congruenz beibehalte, für welches Hr. Bellavitis ohne Noth Coüncidenz setzt. Dafs Hr. Bellavilis jene Gleichung überdies in der Form 
schreibt, ist eine Veränderung der Bezeichnung, welche nicht blofs überflüssig, sondern auch, wenn das Zeichen \| nicht denselben Sinn haben soll wie jedes Gleichheitszeichen, unrichtig genannt werden mufs. (Man sehe meine Ausdehnungslehre, wo sich die Gleichheit planimetrischer Producte, so wie ihre Addition u. s. w. im weitesten Sinne behandelt findet.)

Die wichtigsten Rechnungsregeln, deren ich mich im Folgenden bedienen will, und bei deren Anwendung ein Product sich selbst congruent bleibt, werde ich hier kurz zusammenstellen, indem ich mich dabei auf meine früheren Aufsätze berufe.

Regel 1. Die Stufenzahl eines planimetrischen Products ist der Summe der Stufenzahlen seiner Factoren congruent, in Bezug auf den Modul 3. (Man sehe den entsprechenden Salz für das stereometrische Product in diesem Journal Bd. 49. S. 12.)

Regel 2. Zwei Elemente von gleicher Stufe, welche entweder die ersten Factoren eines Products sind, oder auf einen ersten Factor derselben Stufe folgen, können unter sich vertauscht werden, und geben Null, wenn sie einander congruent sind (Bd. 42. S. 194), z. B.

$$
\begin{aligned}
& a b \equiv b a, A B \equiv B A, a b c \equiv a c b, A B C \equiv A C B, \\
& a a=A A=a b b=A B B=0 .
\end{aligned}
$$

Regel 3. In einem Product nullter Stufe (s. Regel 1) kann man eine schliefsende Klammer weglassen, wenn man zugleich die Ordnung sämmtlicher, frei in der Klammer stehenden ( $d$. h. nicht von einer neuen Klammer umschlossenen) Factoren umkehrt; oder, anders ausgedrückt: Man kann in einem Product nullter Stufe eine Schlufsklammer setzen, wenn man die Ordnung sämmtlicher, frei in die Klammer tretender Factoren umkehrt (Bd. 44. S.5), z. B.

$$
a b C d E f y \equiv \boldsymbol{a}(\boldsymbol{g} f \boldsymbol{E} d \boldsymbol{C b}) \text {. }
$$

'Lusatz. Man kann auch die Ordnung sämmtlicher Factoren eines Products nullter Stufe umkehren (ebend.), z. B.

$$
a b C d E f y \equiv g f E d C b a .
$$

Regel 4. Zwei einander incidente Factoren, welche auf einander folgen, können vertauscht werden (Bd. 42. S. 194), z. B.

$$
a b C \equiv a C b \text {, wenn } b C=0 \text {. }
$$

Regel 5. Ein Factor nullter Stufe (s. Regel 1) kann, wenn er nicht Null ist, weggelassen werden (Bd. 42. S. 194), z. B. (s. Regel 4)

$$
a C b \equiv b \text {, wenn } a C \gtrless 0 \text { ist. }
$$


Regel 6. Wenn in einem Producte zwischen zwei congruenten Elementen ein Element von anderer Stufe steht, so kann man es mit einem der beiden andern zusammen weglassen, falls nicht Incidenz zwischen ihnen Statt findet. $Z$. B.

$$
a b C b \equiv a b, \text { wenn } C b \gtrless 0 \text { ist. }
$$

Beweis. $\quad a b C b \equiv C(a b) b($ Regel 2$) \equiv C b(a b)($ Regel 4$) \equiv a b($ Regel 5$)$, wenn $\boldsymbol{C b} \gtrless 0$.

Regel 7. Wenn ein Product mit 4 Factoren gleicher Stufe beginnt, von denen die zwei letzten von einer Klammer umschlossen sind, und einer der zwei letzten congruent ist mit einem der zwei ersten, so kann man statt aller 4 Factoren einen der beiden congruenten setzen, falls nicht das Product der 3 übrigen Null ist (Ausdehnungslehre. Leipzig 1844. \$. 133), z. B.

$$
\boldsymbol{a b}(\boldsymbol{a c}) \equiv \boldsymbol{a} \text {, wenn } \boldsymbol{a b c} \gtrless \mathbf{0} \text {. }
$$

Denn $a b(a c) \equiv b a(a c)($ Reg. 2) $\equiv b(a c) a$ (Reg. 4) $\equiv a$ (Reg. 5), wenn $a c b \gtrless 0$ ist.

Regel 8. Wenn das Product zweier Elemente, deren eines wieder aus zwei Factoren besteht, gleich Null gesetzt ist, so ist diese Gleichung gleichbedeutend mit dem Vereine zweier anderer Gleichungen, die man aus jenen erhält, wenn man von den letztgenannten Factoren, einmal den einen, dann den andern ausläfst; z. B. die Gleichung

$$
a b C=0
$$

ist, wenn $a b$ ein Element (also nicht Null) ist, gleichbedeutend mit dem Vereine der beiden Gleichungen

$$
a C^{\prime}=0, \quad b C=0 .
$$

Regel 9. Eine Incidenz, welche in Bezug auf $x$ vom $n$ ten Grade ist, bestimmt als geometrischen Ort von $x$ eine Linie nter Ordnung (Bd.31.S.119).

\$. 2 .

Deutung der Gleichung (3.).

Die Gleichung (3.)

$$
x \boldsymbol{A} \cdot \boldsymbol{x b B}, x \boldsymbol{C}=0
$$

ist in Bezug auf $x$ vom dritten Grade; also ist (Reg. 9) der geometrische Ort von $x$ eine Linie dritter Ordnung. Der Ausdruck $x a A$ stellt den Durchschnittspunct der geraden Linien $x a$ und $\boldsymbol{A}$ dar, und da das Product dreier Crelle's Journal f. d. M. Bd. LII. Heft 3. 
Puncte dann, und nur dann Null ist, wenn die drei Puncte in gerader Linie liegen, so enthält obige Gleichung folgenden, schon (Bd. 36.) mitgetheilten, Satz: „Der geometrische Ort eines Puncls, dessen Verbindungslinien mit drei festen Puncten drei feste gerade Linien so schneiden, dafs die 3 Durchschnittspuncte in gerader Linie liegen, ist eine Curve dritter Ordnung."

Die Curve geht, wie ich (Bd. 31. S.125) nachgewiesen habe, durch folgende 9 Puncte:

$$
a, b, c, B C, C A, A B, b c A, c a B, a b C,
$$

die ich beziehlich mit

$$
u, b, c, a_{1}, b_{1}, c_{1}, \alpha, \beta, \gamma
$$

bezeichne. Es haben also die beiden Dreiecke $a b c$ und $a_{1} b_{1} c_{1}$ die Eigenschaft, dafs sich ihre entsprechenden Seiten in den Puncten $\alpha, \beta, \gamma$ treffen; nämlich $b c$ trifft die Seite $b_{1} c_{1}$ oder $A$ in dem Puncte $\alpha$; ca die Seite $c_{1} a_{1}$ oder $B$ in $\beta$, und $a b$ die Seite $a_{1} b_{1}$ oder $C$ in $\gamma$. Folglich ist es, damit sich eine Curve dritter Ordnung mittels der Gleichung (3.) darstellen lasse, nothwendig, dafs man ihr zwei Dreiecke $a b c$ und $a_{1} b_{1} c_{1}$ einschreiben könne, deren entsprechende Seiten sich in 3 Curvenpuncten $\alpha, \beta, \gamma$ schneiden. Um auf rein geometrische Weise zu zeigen, dafs dies bei jeder Curve dritter Ordnung möglich sei, will ich einige Sätze über diese Curven voranschicken.

\section{S. 3 .}

Über den Gang der Curven überhaupt, und der Curven dritter Ordnung insbesondere.

Satz 1. Definition. Ich sage, ein Punct, welcher sich in einer Ebene bewegt, bewege sich stetig, wenn die gerade Linie, welche von irgend einem Puncte aufserhalb der Ebene nach ihm gezogen wird, bei jener Bewegung niemals aus einer Lage unmittelbar in eine andere übergeht, welche mit jener einen endlichen Winkel bildet.

Beinerkung. So lange der Punct in endlicher Entfernung bleibt, läfst sich seine stetige Bewegung auch dadurch bestimmen, dafs er bei jener Bewegung niemals aus einer Lage in eine andere übergeht, welche um eine endliche Strecke von jener entfernt liegt.

Satz 2. Definition. Wenn ein Punct von einer beliebigen Lage aus sich stetig so bewegt, dafs er keinen Punct seiner früheren Bahn berührt, bis er wieder in seine ursprüngliche Lage zurückkommt, so nenne ich diese Bahn einen Zug, und sage, der Punct habe diesen Zug einmal durchlaufen. 
Bemerkung. In diesem Sinne wird man z. B. sagen können, dafs jeder Kegelschnilt, der nicht in zwei gerade Linien zerfällt, aus Einem Zuge bestehe.

Sat: 3. Jede Projection eines Zuges ist wieder ein Zug.

Satz 4. Jede Curve dritter Ordnung besteht entweder aus zwei Zügen, oder aus nur einem Zuge, oder aus einem Zuge und einem isolirten Punct, und zwar sind die drei reellen Wendepuncte jedesmal in einem Zuge enthalten.

Beweis. Es geht dies am deutlichsten aus den 5 von Newton aufgestellten divergirenden Parabeln hervor, durch deren Projection alle Curven dritter Ordnung erzeugt werden können (Newt. Enumeratio linearum tertii ordinis pag. 92, 93). Die Curve mit einem Kreuzpuncte mufs nach der aufgestellten Definition als aus zwei Zügen bestehend angesehen werden, welche in dem Kreuzpuncte zusammenstofsen. Eine in gerade Linien zerfallende Linie höherer Ordnung rechne ich nicht zu den Curven.

Satz 5. Wenn eine gerade Linie, die sich um einen festen Punct stetig bewegt, eine algebraische Curve schneidet, so kann die stetige Fortbewegung der Durchschnittspuncte nur gleichzeitig bei zweien dieser Puncte aufhören.

Bemerkung. Alle diese Sätze sind entweder bekannt, oder unmittelbar einleuchtend.

Satz 6. Zieht man von irgend einem festen Puncte einer Curve dritter Ordnung, der aber nicht ein Doppelpunct ist, eine Gerade nach einem beweglichen Puncte, welcher einen Zug der Curve einmal durchläuft, so durchläuft auch der dritte Punct, in welchem jene Gerade die Curve trifft, einen Zug derselben einmal.

Beweis. Es sei $a$ der feste Punct, $p$ der bewegliche, welcher einen Zug der Curve einmal durchläuft, und $q$ der dritte Durchschnittspunct von ap mit der Curve. Zu zeigen ist, dafs auch $q$ einen Zug der Curve einmal durchläuft. Erstens kann während jener Bewegung $q$ nicht unbeweglich bleiben, weil sonst $a$ ein Doppelpunct wäre; zweitens kann aber $q$ nie aufhören sich stetig zu bewegen, weil sonst, nach (Satz 5), auch einer der beiden andern Puncte $p$ oder $a$ aufhören müfste, sich stetig zu bewegen. Für $p$ ist dies gegen die Annahme; für $a$ ist es gleichfalls nicht möglich, da sich $a$ nach der Annahme gar nicht bewegt. Drittens kann aber auch $q$ nicht einen früheren Punct seiner Bahn, etwa den Punct $q^{\prime}$, wieder berühren; denn es sei 
$p^{\prime}$ der Punct, in welchem $a q^{\prime}$ die Curve aufser $a$ und $q^{\prime}$ trifft, so ist $p^{\prime}$ der Punct, in welchem sich beidemale $p$ befunden haben müfste, während $q$ sich in $q^{\prime}$ befindet; also müfste auch $p$ und $\boldsymbol{p}^{\prime}$ wieder einen Punct seiner früheren Bahn berührt haben; gegen die Annahme. Endlich, wenn $p$ wieder zu seiner Anfangslage zurückkehrt, so kehrt auch q zu ihr zurück; also durchläuft $q$ während jener Bewegung die Curve einmal.

Satz 7. Die Anzahl der Puncte, in welchen eine algebraische Curve den Umfang einer geschlossenen Figur schneidet, ist stets eine gerade.

Beweis. Da jeder $Z_{w}$ eig einer algebraischen Curve entweder in sich geschlossen ist, oder nach beiden Seiten ins Unendliche hin sich erstreckt, so mufs jeder Zweig der in das Innere der Figur hineingeht, auch wieder aus demselben herausgelangen; mithin mufs die Anzahl der Curvenstücke, welche den Umfang schneiden, gerade sein; folglich auch die Anzahl der Puncte, in welchen die Curve den Umfang schneidet, indem, wenn $m$ Curvenzweige durch denselben Punct des Umfangs gehen, dieser als ein mfacher Punct gerechnet wird.

S. 4 .

Entsprechende Dreiecke, welche einer Curve dritter Ordnung eingeschrieben sind.

Wenn die Richtung, in welcher eine gerade Linie in einer Ebene durchlaufen wird, bekannt ist, so ist dadurch auch ihre rechte oder linke Seite bestimmt. Ich sage, ein Punct $c$ liege von der geraden Linie $a b$ aus nach rechts, wenn man, um auf zwei geradlinigen Wegen von $a$ über $b$ nach $c$ zu gelangen, nach rechls hin abbiegen mufs.

Salz 8. Wenn eine gerade Linie $(p q)$ sich um einen festen Punct $c$ dreht, und zwei Puncte in ihr ( $p$ und $q$ ) sich in zwei festen Geraden ( $\boldsymbol{A}$ und $\boldsymbol{B}$ ) bewegen, welche während der Bewegung nie mit jener beweglichen geraden Linie ( $p y)$ zusammenfallen: so bewegen sich beide Puncte ( $\boldsymbol{p}$ und $q$ ), von der beweglichen Linie aus, nach derselben Seite, wenn der Drehpunct (c) au/serhalb dieser Puncte liegt, und nach entgegengesetzter, wenn innerhalb.

Satz 9. Wenn die Schenkel eines Winkels (cqa) sich um zwei feste Puncte $(c$ und $a)$ drehen, und der Scheitelpunct desselben $(q)$ sich in einer festen geraden Linie $(B)$ bewegt, welche während der Bewegung mit keinem der Schenkel zusammenfällt: so bewegt sich der Scheitelpunct $(q)$, von den beiden Schenkeln (cq und $a q$ ) aus, nach derselben Seite, wenn die !gerade 
Linie (B) nicht in den Winkel hineingeht, und nach entgegengesetzter Seite, wenn sie hineingeht.

Nach diesen vorbereitenden Sätzen, von welchen die beiden letzten keines Beweises bedürfen, schreite ich nun zu dem Hauptsatze:

Satz 10. Zu jedem Dreiecke abc, welches einer Curve dritter Ordnung eingeschrieben ist, und dessen Seiten nicht Tangenten sind, giebt es ein, aber auch nur ein entsprechendes-Dreieck $a_{1} b_{1} c_{1}$, dessen Seiten die entsprechenden des ersteren in Puncten der Curve schneiden, und von dessen Ecken jede mit der entsprechenden in demselben Curvenzuge liegt.

Beweis. Es seien $\alpha, \beta, \gamma$ die Puncte, in welchen die Seiten $b c, c a$, $a b$, beziehlich, die Curve zum drittenmale schneiden, und $\boldsymbol{A}, \boldsymbol{B}, \boldsymbol{C}$ seien die Tangenten der Curve in den Puncten $a, b, c$. Nun bewege sich von $a$ aus ein Punct $p$ auf der Curve, also von $a$ aus in der Richtung der Tangente $\boldsymbol{A}$. Man ziehe die gerade Linie $\gamma p$, welche die Curve zum drittenmale in $q$ treffen mag, so bewegt sich $q$ von $b$ aus in der Richtung der Tangente $\boldsymbol{B}$. Ferner ziehe man die gerade Linie $\alpha q$, welche die Curve zum drittenmale in $r$ treffen mag, so bewegt sich $r$, von $c$ aus in der Richtung der Tangente $C$. Endlich ziehe man die gerade Linie $\beta r$, welche die Curve zum drittenmale in $\mu_{1}$ treffen mag, so bewegt sich $p_{1}$ von $a$ aus in der Richtung der Tangente $\boldsymbol{A}$. Auch $\boldsymbol{p}$ bewegte sich von demselben Puncte $a$ aus in der Richtung derselben Tangente $\boldsymbol{A}$. Es läfst sich aber leicht zeigen, dafs $p$ und $p_{1}$ von $a$ aus nach entgegengesetzten Seiten sich bewegen. In der That mögen $m$ von den drei Puncten $\alpha, \beta, \gamma$ innerhalb der Seiten des Dreiecks abc liegen, und $n$ von den 3 Tangenten $\boldsymbol{A}, \boldsymbol{B}, \boldsymbol{C}$ nicht in das Dreieck $\boldsymbol{a b c}$ hineingehen, so werden, da der Annahme gemäfs die Seiten des Dreiecks abc keine Tangenten sind, $3-n$ von den Tangenten $\boldsymbol{A}, \boldsymbol{B}, \boldsymbol{C}$ in das Dreieck hineingehen; ebenso aber diejenigen in Curventheile, welche die (unverlängerten) Seiten des Dreiecks treffen. Also wird, nach (Satz 7), $m+3-n$ eine gerade Zahl sein, folglich $m-n$ eine ungerade, mithin auch $m+n$ eine ungerade Zahl sein.

Betrachtet man nun der Reihe nach die Seiten (rechte oder linke), nach welchen sich $p$ und $q$ von $a b$ aus, $q$ und $r$ von $b c$ aus, $r$ und $p_{1}$ von $c a$ aus und $p_{1}$ von $a b$ aus bewegen, zeigt sich klar, nach (Salz 8), dafs $p$ und $q$ von $a b$ aus dann, und nur dann, nach entgegengeselzten Seiten sich bewegen, wenn $\gamma$ innerhalb der Seite $a b$ liegt; dasselbe gilt für die Bewegung von $q$ und $r$ von $b c$ aus, und für die von $r$ und $p_{1}$ von $c a$ aus. Ferner wird $q$ (nach Satz 9) von $a b$ und $b c$ aus sich dann, und nur dann, nach 
entgegengeselzten Seiten bewegen, wenn die Tangente $\boldsymbol{B}$ nicht in das Dreieck hineingeht; und dasselbe gilt für die Bewegung des Punctes $r$ von $b c$ und $c a$ aus, so wie des Punctes $p_{1}$ von $c a$ und $a b$ aus. Folglich wird sich die Seite, nach welcher die Bewegung in den $\boldsymbol{\gamma}$ oben genannten Fällen erfolgt, $\boldsymbol{m}+\boldsymbol{n}$ mal umkehren, also, da $m+n$ ungerade ist, im siebenten Falle entgegengesetst sein, wie im ersten; d. h. $p_{1}$ bewegt sich von $a b$ aus nach entgegengesetzter Seite wie $p$.

Läfst man nun den Punct $p$ von $a$ aus den ganzen Curven-Zug, in welchem a liegt, einmal durchlaufen, so durchläuft, nach (Satz 6) der Punct $q$ gleichfalls einen Zug der Curve einmal, und weil $q$, so auch $r$, und weil $r$, so auch $p_{1}$. Folglich durchlaufen $p$ und $p_{1}$ von $a$ aus nach entgegengesetzten Seiten den Zug, in welchem $a$ liegt, einmal, müssen sich also in einem zweiten Puncte dieses Zuges begegnen, aber auch in keinem dritten. Es sei $a_{1}$ dieser Punct, und mögen $q$ und $r$, während $p$ in $a_{1}$ übergeht, in $b_{1}$ und $c_{1}$ übergehen, so ist $a_{1} b_{1} c_{1}$ das entsprechende Dreieck, dessen Seiten die entsprechenden des Dreiecks $a b c$ in den Curvenpuncten $\alpha, \beta, \gamma$ schneiden, und dessen Ecken mit den entsprechenden in denselben Curvenzügen liegen. Aufser ihm giebt es kein anderes Dreieck dieser Art, q. d. e.

Zusatz. Wenn die drei Puncte $(\alpha, \beta, \gamma)$, in welchen die Seiten eines Dreiecks $(\boldsymbol{a b c})$ die Curve zum drittenmale schneiden, in gerader Linie liegen, und jeder dieser Puncte mit der gegenüberliegenden Ecke ( $\alpha$ mit $a$, $\beta$ mit $b, \gamma$ mit $c$ ) in demselben Curvenzuge liegt, so streckt sich das, jenem Dreieck entsprechende in die gerade Linie, welche jene drei Puncte $(\alpha, \beta, \gamma)$ verbindet, aus; und es giebt dann aufserdem kein Dreieck, welches dem gegebenen $(a l b c)$ in der genannten Weise entspricht.

S. 5.

Allgemeinheit der Curven dritter Ordnung, welche durch die Gleichung (3.) dargestellt werden.

Es sei eine beliebige Curve dritter Ordnung gegeben. Um sie auf die Gleichung (3.) zurückzuführen, zeichne man in demjenigen Zuge derselben, der die Wendepuncte enthält, ein beliebiges Dreieck, desssen Seiten jedoch nicht Tangenten sind; dann müssen die dritten Durchşchnittspuncte der Seiten und der Curve in demselben Zuge liegen. Sollten diese 3 Durchschnittspuncte etwa in gerader Linie liegen, so ist nach obigem Zusatze das angenommene Dreieck das einzige demselben Zuge eingeschriebene, dessen Seiten 
durch jene 3 Durchschnittspuncte gehen. Verändert man also in dem genannten Zuge das Dreieck auf beliebige Weise, jedoch so, dafs zwei seiner Seiten durch zwei jener Durchschnittspuncte gehen, so kann die dritte Seite nicht den dritten Durchschnittspunct treffen.

Hat man nun das Dreieck so angenommen, dafs seine Seiten nicht Tangenten sind, so hat man ir jedem Falle ein Dreieck abc erlangt, dessen Seiten, ohne Tangenten zu sein, die Curve zum drittenmale in drei Puncten $\alpha, \beta, \gamma$ schneiden, welche nicht in gerader Linie liegen.

Jetzt construire man das entsprechende Dreieck $a_{1} b_{1} c_{1}$, dessen Seiten sich mit den entsprechenden des Dreiecks alsc in den Curvenpuncten $\alpha, \beta, \gamma$

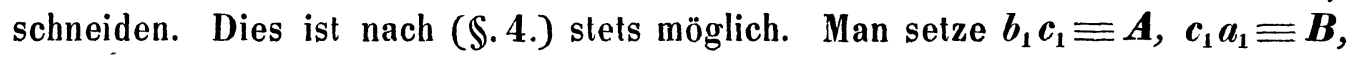
$a_{1} b_{1} \equiv C$, so ist der geometrische Ort eines Punctes $x$, welcher der Gleichung

$$
\text { (3.) } \quad x a A \cdot x b B \cdot x c C=0
$$

genügt, eine Curve dritter Ordnung, welche mit der gegebenen die 9 Puncte $a, b, c, a_{1}, b_{1}, c_{1}, \alpha, \beta, \gamma$ gemein hat. Es bleibt also nur noch zu zeigen, dafs diese 9 Puncte von der Art sind, dafs durch sie eine Curve dritter Ordnung vollkommen bestimmt wird, d. h. dafs zwei Curven dritter Ordnung, deren jede durch diese 9 Puncte geht, mit einander zusammenfallen.

Zu dem Ende berufe ich mich auf folgenden allgemein bekannten Satz: "Wenn zwei Linien 3ter (nter) Ordnung sich in 9 (in $n^{2}$ ) aber auch „nicht in mehr Puncten treffen, und man nimmt zu $8\left(\mathrm{zu} \frac{1}{2} n(n+3)-1\right)$ ,derselben einen Punct hinzu, welcher nicht jenen beiden Linien dritter „(nter) Ordnung gemein ist, so wird durch diese 9 (durch diese $\frac{1}{2} n(n+3)$ ) „Puncle eine Linie dritter (nter) Ordnung unter allen Umständen voll"kommen bestimmt."

Legt man nun durch die drei Puncte $b, c, \alpha$ eine Gerade, ferner eine zweite Gerade durch die 3 Puncte $c_{1}, a_{1}, \beta$, und eine dritte Gerade durch die 3 Puncte $a, \gamma, b$, so bildet der Verein dieser 3 Geraden, eine Linie dritter Ordnung, welche mit der gegebenen Curve dritter Ordnung, aufser den genannten Puncten, unter denen $b$, als Doppelpunct der einen, zweimal gerechnet werden mufs, keinen Punct weiter gemein hat, weil jede Curve dritter Ordnung von einer geraden Linie in nicht mehr als 3 Puncten getroffen werden kann. Der Punct $b_{1}$ kann nun in keiner jener drei Geraden liegen, weil sonst diese Gerade die Curve in 4 Puncten treffen würde: also ist $b_{1}$ nicht beiden Linien dritter Ordnung gemein, während die übrigen 8 Puncte 
$a, b, c, a_{1}, c_{1}, \alpha, \beta, \gamma$ beiden gemein sind. Somit wird nach dem angeführten Salze durch die 9 Puncte $a, b, c, a_{1}, b_{1}, c_{1}, \alpha, \beta, \gamma$ eine Linie dritter Ordnung vollkommen bestimmt; d. h. zwei Linien dritter Ordnung, welche diese 9 Puncte gemein haben, sind identisch; also ist die gegebene Curve mit dem durch die obige Gleichung (3.) bestimmten geometrischen Orte des Puncls $x$ identisch; folglich läfst sich jede Curve dritter Ordnung durch eine Gleichung von der Form (3.) darstellen. D. h.

Jede Curve dritter Ordnung lä/st sich als geometrischer Ort eines Punctes erzeugen, dessen Verbindungslinien mit drei festen Puncten drei feste gerade Linien in drei Puncten schneiden, die in gerader Linie liegen; und zwar kommt es zu dem Ende nur darauf an, das Dreieck der festen Puncte und das der festen Geraden so anzunehmen, da/s sie der Curve eingeschrieben sind, und da/s die entsprechenden Seiten beider auf der Curve sich begegnen.

Bemerkung. Hr. Bellavilis behauptet, dafs sich durch die Gleichung (3.) nur solche Curven dritter Ordnung darstellen lassen, welche schon durch 6 beliebige Puncte bestimmt sind, d. h. welche schon bestimmt sind, wenn nur 6 Puncte gegeben sind, durch welche sie gehen sollen. An diesem Resultate hätte Hr. Bellavitis schon um deswillen sich stofsen sollen, weil es keine Curve dritter Ordnung giebt, die durch 6 Puncte auf lineale Weise bestimmt wird.

Die Art, wie er zu seinem Resultate gelangt, ist im Wesentlichen folgende: Wenn nämlich 6 Puncte $a, b, c$ und $a_{1}, b_{1}, c_{1}$ gegeben sind, so sind auch die Linien $\boldsymbol{A}, \boldsymbol{B}, \boldsymbol{C}$ als Seiten des Dreiecks $a, b, c$, also alle constanten Elemente der Gleichung (3.); mithin auch die durch sie dargestellte Curve dritter Ordnung bestimmt, und folglich (so schliefst Hr. Bellavitis weiter) ist diese Curve dritter Ordnung schon bestimmt, wenn 6 Puncte $a, b, c$, $a_{1}, b_{1}, c_{1}$ gegeben sind, welche in ihr liegen sollen; und dies ist der Fehlschlufs. Nicht dadurch schon ist jene Curve bestimmt, dafs die genannten 6 Puncte in ihr liegen sollen, sondern erst dadurch, dafs die 6 Puncte auch in der verlangten Weise in ihr liegen sollen, nämlich in der Weise, dafs die entsprechen Dreiecke $a b c$ und $a_{1} b_{1} c_{1}$ sich auf der Curve begegnen; wie ich dies auch schon in der oben angeführten Abhandlung (Bd. 36. S. 180) bemerkt habe. Also, so wenig man daraus, dafs ein Kreis durch die Endpuncte eines Durchmessers bestimmt wird, schliefsen darf, dafs der Kreis schon durch zwei beliebige Puncte bestimmt werde, ebenso wenig kann jener Schlufs Geltung 
haben. Ganz dieselben Fehlschlüsse wendet Hr. Bellavitis an, um die Allgemeingültigkeit der beiden andern Constructionsmethoden zu bestreiten; wobei er auf die von mir gegebenen Beweise der Allgemeinheit keine Rücksicht nimmt.

\section{\$. 6 .}

Zusammenhang zwischen den verschiedenen linealen Erzeugungsweisen einer Curve dritter Ordnung.

Es wird dieser Zusammenhang am klarsten sich ergeben, wenn ich zeige, wie sich alle andern linealen Erzeugungsweisen auf eine derselben, zu welcher ich die durch die Gleichung (2.) dargestellte nehme, direct zurückführen lassen; nämlich in der Art, dafs man, wenn man die gegebene Erzeugungsweise gleichfalls durch eine lineale Gleichung darstellt, aus den constanten Elementen dieser Gleichung stets solche Elemente ableiten kann, welche in die Gleichung (2.) gesetzt, diese der gegebenen gleichbedeutend machen. Nun habe ich in dem angeführten Aufsatze (Bd. 36. S. 178) folgenden Satz bewiesen:

„Der geometrische Ort des Punctes $x$, der durch die Gleichung

$$
x a A a_{1}, x b B b_{1}, x c=0
$$

bestimmt wird, ist eine Linie dritter Ordnung, welche durch folgende 9 Puncte geht: erstens durch $a, b, c$, zweitens durch die Ecken eines Vierecks, von welchem zwei einander gegenüberliegende Seiten in den geraden Linien $\boldsymbol{a a _ { 1 }}$ und $\boldsymbol{A}$, und die beiden andern in den geraden Linien $b b_{1}$ und $B$ liegen, und endlich durch die beiden Puncte, in welchen $a_{1} c$ die Seite $A$, und $b_{1} c$ die Seite $B$ schneiden,"

und den umgekehrten Satz:

"Werden in einer Seite eines Vierecks zwei beliebige Puncte $a$ und $a_{1}$ angenommen, während die gegenüberliegende Seite in der geraden Linie $\boldsymbol{A}$ liegt; sind ferner in einer dritten Seite zwei beliebige Puncte $b$ und $b_{1}$ angenommen, während die gegenüberliegende Seite in der geraden Linie $\boldsymbol{B}$ liegt, und ist $c$ ein beliebiger Punct, der nicht in den Vierecksseiten liegt, so ist

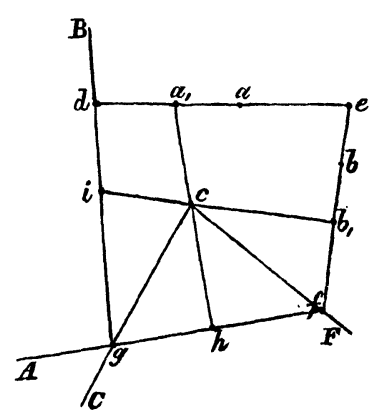

$$
x a A a_{1}, x b B b_{1}, x c=0
$$

die Gleichung derjenigen Curve dritter Ordnung, welche durch die Ecken Crelle's Journal f. d. M. Bd. LII. Heft 3. 
des Vierecks und durch den Punct $c$ geht, und die Vierecksseiten aufserdem in den Puncten $a, b$ und in denjenigen zwei Puncten triff, in welchen die Geraden $a_{1} c$ und $b_{1} c$ die gegenüberliegenden Seiten $\boldsymbol{A}$ und $\boldsymbol{B}$ schneiden; und zwar giebt es aulser dieser Curve keine andere Curve dritter Ordnung, welche durch die bezeichneten 9 Puncte ginge." (S. Fig.)

Jede lineale Erzeugung einer Curve dritter Ordnung läfst sich nun (s. Bd. 42. S. 190) durch ein gleich Null gesetztes Product darstellen, welches mit dem construirenden Puncte $x$ beginnt und schliefst und aufserdem eine Reihe abwechselnder Puncte und gerader Linien als Factoren enthält, die mit einem Puncte beginnt und schliefst, während einer der übrigen Factoren der Reihe von dem Puncte $x$ im ersten Grade abhangt, also in der Form

$$
\text { (4.) } \quad x d \mathrm{~A} f x=0 \text {, }
$$

wo A eine Reihe abwechselnder gerader Linien und Puncte ist, von denen einer noch den Punct $\boldsymbol{x}$ als Factor enthält.

Es seien $e$ und $g$ zwei beliebige Puncte der Curve (4.), von der Art, dafs keine 3 der 4 Puncte $d, e, f, g$ in gerader Linie liegen. Durch Construction läfst sich leicht der dritte Punct finden, in welchem jede Seite des Vierecks defg die Curve schneiden mufs. Wird z. B. der dritte Punct $x$ gesucht, in welchem die Seite ed die Curve schneidet, so fält die Gerade $x d$ mit ed zusammen und die Gleichung (4.) verwandelt sich in

$$
\text { (5.) } \quad \text { edA } f x=0 \text {. }
$$

Da dieselben nur noch vom zweiten Grade in Bezug auf $x$ ist, so ist der geometrische Ort von $x$ ein Kegelschnitt. In diesem Kegelschnitte liegt (nach Regel 2) der Punct $f$; ferner aber auch der Punct $e$, indem $e$ der Annahme zufolge ein Punct der Curve (4.) ist, also, statt $x$ gesetzt, der Gleichung (4.), und mithin auch der Gleichung (5.) genügt.

Es lassen sich nun beliebig viele neue Puncte dieses Kegelschnittes lineal construiren. Es seien $k, l, m$ drei neue Puncte desselben. Bildet man das Sechseck xeklmf; so liegen, nach dem Pascal'schen Satze, die Puncte $x e . l m$, ek.mf; $k l . f x$ in einer geraden Linie, d. h. es ist

$$
\text { (6.) } \quad x e(l m)(e k . m f)(k l) f x=0
$$

die Gleichung des Kegelschnitts, der durch die 5 Puncte $e, k, l, m, f$ geht, also mit dem Kegelschnitte (5.) identisch ist. Es ist demnach der gesuchte Punct $x$ derjenige, in welchem die Gerade ed den Kegelschnitt aufser e noch 
triff. Da $x$ in $e d$ liegt, und nicht mit $e$ identisch ist; so ist die Gerade $x e$ mit de identisch, und die Gleichung (6.) verwandelt sich in

$$
\operatorname{de}(\mathrm{lm})(\mathrm{ek} . \mathrm{mf})(\boldsymbol{k} l) f x=0 ;
$$

d. h. der Punct $x$ liegt in der Geraden $\operatorname{de}(\boldsymbol{l m})(e k . m f)(k l) f$; er liegt aber auch in der Geraden de, also im Durchschnitt beider, d. h. wenn man diesen Punct jetzt mit $a$ bezeichnet, so ist

$$
\text { (7.) } \quad a \equiv \operatorname{de}(\mathrm{lm})(\mathrm{ek} . \mathrm{mf})(\boldsymbol{k l}) \boldsymbol{f}(\mathrm{de}) \text {. }
$$

Ganz auf dieselbe Weise finden sich die dritten Puncte, in welchen die Seiten $e f, f g, g d$ die Curve (4.) schneiden. Sie mögen beziehlich durch $b$, $h, i$ bezeichnet werden (s. Fig.). Dann ist, wie bekannt, auch der Durchschnitt von ah und bi ein Punct der Curve. Endlich sei $c$ ein beliebiger zehnter Punct der Curve (4.), und es werde $f g$ durch $\boldsymbol{A}$, $d g$ durch $B$, der Durchschnitt von $h c$ und de durch $a_{1}$ und der Durchschnitt von ic und ef durch $b_{1}$ bezeichnet; dann ist nach dem obigen Satze die Gleichung

$$
\text { (8.) } \quad x a A a_{1}, x b B b_{1}, x c=0
$$

die einer Curve dritter Ordnung, welche durch die 9 Puncte $a \ldots i$ geht; und zwar ist diese Curve, nach jenem Satze, die einzige dritter Ordnung, welche durch die 9 Puncte geht. Aber auch die Curve (4.) geht durch diese 9 Puncte, also ist die Curve (4.) mit der (8.) identisch; mithin ist auch die Gleichung (4.) mit der Gleichung (8.) gleichbedeutend; und die Aufgabe der Umwandlung ist gelöset.

Als Beispiel der Umwandlung nehme ich die von Hrn. Bellavilis aufgestellte Gleichung,

$$
\text { (9.) } x e D_{p} E d F(x f B) . x d C=0 \text {, }
$$

an, in welcher noch $F f$ und $B d$ gleich Null gesetzt sind (s. Fig.), und welche nach ihm die erste allgemeine Auflösung des Problems der linealen Erzeugung der Curven dritter Ordnung darstellen soll; und zwar diejenige, welche Chasles in dem angeführten Aufsatze angegeben hat.

Es läfst sich diese Gleichung (Regel 3) wie folgt schreiben:

$$
x e D_{p} \boldsymbol{E d F}(x d C) \boldsymbol{B} f x=0 \text {; }
$$

wobei sich annehmen läfst, dafs von den constanten Factoren keine zwei auf einander folgende incident sind, weil sonst (Bd. 42. S.197) die Linie in eine gerade Linie und einen Kegelschnitt zerfallen würde. Puncte jener Curve sind (Regel 2) $d$, e, $f$. Ferner ist auch $g \equiv B C$ ein Punct derselben. Denn dann 
ist $g$ mit $B$ und $C$ incident; also verwandeln sich dann $x d C$ und $x f B$, wenn sie nicht selbst Null sind (Regel $2,4,5$ ), beide in $g$, folglich wird $\boldsymbol{x} d \boldsymbol{C}(\boldsymbol{x} \boldsymbol{F} \boldsymbol{B})=0$ (Reg. 2). Um den dritten Punct $a$, in welchem de die Curve schneidet zu finden, mufs man in obige Gleichung $a$ statt $x$ und statt $a e$ und ad das mit ihnen identische de setzen; dann wird $\operatorname{deD} \boldsymbol{E d} \boldsymbol{E}(\operatorname{deC}) \boldsymbol{B} f a=0$, also, da $a$ auch in de liegt, zu

$$
a \equiv \operatorname{deD} p E d F(d e C) B f(d e)
$$

und eben so findet sich für den dritten Punct $b$, in welchem ef die Curve trifft:

$$
\boldsymbol{b} \equiv \boldsymbol{e} \boldsymbol{D}_{\boldsymbol{p}} \boldsymbol{E d F}(\boldsymbol{e} \boldsymbol{C} \boldsymbol{B}) \boldsymbol{C d}(\boldsymbol{e} \boldsymbol{f})
$$

Es mögen jetzt die dritten Puncte $h$ und $i$ gesucht werden, in denen $f g$ und $g d$ die Curve treffen. Setzt man, um den dritten Durchschnittspunct $h$ in $f g$ zu finden, $h$ in die Gleichung (9.) statt $x$, so erhält man, da $h$ in $f y$ liegt, $x f \equiv h f \equiv f g$; und da $g$ mit $B$, wie mit $C$ incident ist, so ergiebt sich $x f B \equiv f g B \equiv g$ (Reg. 4, 5) und $x d C(x f B) \equiv x d C g \equiv x d g . C$ (Reg. 4). Sollte $x d g$ Null sein, so müfste $x$ in $d g$, wie in $f g$, also in $g$ liegen. Um also den dritten Durchschnittspunct zu finden, mufs man $x d g$ ungleich Null annehmen; dann erhält man $x d C .(x f B) \equiv C$ (Reg. 5), und somit heDpEdFC=0, oder umgekehrt (Reg. 3) FCdEpDeh $=0$; folglich, da $h$ auch in fy liegt,

und ebenso

$$
h \equiv \boldsymbol{F C d E p D e}(f g) \text {, }
$$

$$
\boldsymbol{i} \equiv \boldsymbol{B F d E p D e}(\boldsymbol{g d}) \text {. }
$$

Ferner ist auch $\boldsymbol{C F}$ ein Punct der Curve. Denn setzt man $\boldsymbol{C F}$ statt $x$, so wird $x d C \equiv F C d C \equiv F C$ (Reg. 6), und $x f B$ wird $\equiv C F f B \equiv C f . F B$ (Reg. 4), da $f$ nach der Annahme mit $\boldsymbol{F}$ incident ist. Ist nun $\boldsymbol{C} f$ nicht Null, so kann man es nach (Reg. 5) weglassen. Also wird $x \boldsymbol{B} B$ entweder zu Null oder zu $\equiv \boldsymbol{F B}$; also $x \boldsymbol{F}$.(xcD) entweder zu Null oder zu $\equiv \boldsymbol{F C}(\boldsymbol{F B}) \equiv \boldsymbol{F}$ (Reg. 2, 4, 5). Somit erhält man dann

$$
x e D_{p} E d F(x f B) \cdot x c D \equiv x e D_{p} E d F F=0 \text { (Reg. 2), }
$$

also ist $\boldsymbol{x} \equiv \boldsymbol{F C}$ ein Punct der Curve. Dieser werde $\equiv \boldsymbol{c}$ gesetzt. Wird nun, wie oben, $g f \equiv A$, $h c . d e \equiv a_{1}$, ic.ef $=b_{1}$ gesetzt, so ist die Gleichung

$$
x a A a_{1}, x b B b_{1}, x c=0
$$

gleichbedeutend mit der Gleichung (9.). Also folgt Nachstehendes: 
„Die Gleichung

$x e D p E d F(x f B) \cdot x d C=0, \quad$ mit $\quad F f=B d=0$,

ist gleichbedeutend mit der Gleichung

$$
x a A a_{1} . x b B b_{1} . x c=0,
$$

wo $a \equiv \operatorname{deD} p \boldsymbol{E d F}(\operatorname{deC}) \boldsymbol{B} f(d e), b \equiv e f D_{p} E d F(e f B) C d(e f), c \equiv F C$, $A \equiv B C f, a_{1} \equiv c d E p D e A c(d e), b_{1} \equiv B F d E p D e B c(e f)$ ist."

\$. 7 .

Lineale Erzeugung einer Curve dritter Ordnung aus 9 beliebigen Puncten.

Entwurf der Auflösung. Jede lineale Erzeugung einer Curve dritter Ordnung kann durch eine Gleichung von der Form

$$
\boldsymbol{P Q R}=\mathbf{0}
$$

dargestellt werden, in welcher $\boldsymbol{P}, \boldsymbol{Q}, \boldsymbol{R}$ entweder 3 gerade Linien, oder 3 Puncte sind, die in beiden Fällen in Form von Producten vorkommen, deren jedes den construirenden Punct $x$ einmal als Factor enthält.

Es seien nun $a, b, \ldots i$ die 9 Puncte, durch welche die durch jene Gleichung darzustellende Curve gehen soll. Es giebt stets 3 Puncte, die statt $x$ gesetzt ein solches Product $\boldsymbol{P Q}$ zu Null machen; und die constanten Factoren in $\boldsymbol{P}$ und $\boldsymbol{Q}$ lassen sich so annehmen, dafs $a, b, c$ diese 3 Puncte sind. Ferner wird $\boldsymbol{R}$ durch den Punct $\boldsymbol{d}$ zu Null gemacht, wenn man dem $\boldsymbol{R}$ die Form $x d \ldots$ giebt. Setzt man nun in $\boldsymbol{P Q}$ statt $x$ nach und nach die Puncte $e \ldots i$, und ebenso in $x d$, so stellt $\boldsymbol{P Q}$ nach und nach 5 Elemente dar, und $x d$ einen Büschel von 5 Strahlen. Es lassen sich aber im Allgemeinen aus 5 gegebenen Elementen 5 gegebene Strahlen eines Strahlbüschels projectivisch, d. h. durch fortschreitende Multiplication mit einer Reihe abwechselnder Puncte und Linien ableiten; und zwar sind diese Puncte und Linien lineal construirbar. Es sei A diese Reihe fortschreitender Factoren, so stellt das Product PQA eine gerade Linie dar, welche mit der geraden Linie $x d$, sobald man statt $x$ irgend einen der 5 Puncte $e \ldots i$ setzt, zusammenfällt. Also ist für diese 5 Puncte:

$$
\text { (10.) } \quad P Q A x=0 \text {. }
$$

Aber auch für die 4 Puncte $a, b, c, d$ wird diese Gleichung erfüllt: für $a, b, c$, da für sie das Product $P Q$ Null ist, und für $d$, da $P Q A$, wenn es nicht Null ist, eine durch $d$ gehende gerade Linie darstellt, also PQAd stets Null ist. Die Gleichung (10.) stellt nun aber eine Curve dritter Ordnung 
als Ort von $x$ dar, und da die 9 Puncte $a \ldots i$, statt $x$ gesetzt, jener Gleichung genĩgen, so geht die Curve durch die gegebenen neun Puncte, und die Aufgabe ist gelöset.

\section{S. 8 .}

\section{Fortsetzung.}

Um eine specielle Lösung der Aufgabe zu geben, will ich annehmen, es solle die lineale Construction der Curve die durch die 9 Puncte $a \ldots i$ gehen soll, durch eine Gleichung von der Form (2.) ausgedrückt werden, zu welcher jedoch, um die Lösung zu vereinfachen, zunächst noch 2 Factoren $k$ und $C$ hinzugefügt werden mögen, so dafs sie die Form

(11.) $\quad x a A a_{1}, x b B k C b_{1}, x c=0$

annimmt. Die 3 Factoren können (Regel 2) beliebig umgeordnet werden. Setzt man

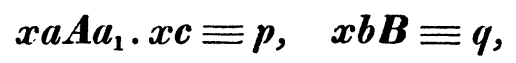

so nimmt die Gleichung die Form $p\left(q k C b_{1}\right)=0$, oder (Reg. 3)

$$
\text { (12.) } \quad \mu b_{1} C k q=0
$$

an. Der Ausdruck $p$ wird Null für $x \equiv a$ und $c$, weil dann zwei auf einander folgende Factoren congruent werden (Reg. 2). Damit nun $p$ auch noch für einen andern der Puncte $a \ldots i$, z. B. für $d$ zu Null werde, und für einen vierten und fünften Punct $e$ und $f$ sich möglichst vereinfache, setze man

$$
\boldsymbol{A} \equiv \mathrm{de}, \quad a_{1} \equiv \text { af.cd. }
$$

Und zwar nehme man die Puncte $a, c, d, e, f$ so an, dafs keine 3 derselben in gerader Linie liegen. Dann wird erstens für $x \equiv d$ der Ausdruck $x a A a_{1} \equiv d a(d e) a_{1} \equiv d a_{1}$ (Reg. 7) $\equiv a_{1} d \equiv a f(c d) d \equiv c d$ (Reg. 4, 5); also $p \equiv x a A a_{1} . x c \equiv c d . c d=0$ (Reg. 2). Ferner für $x \equiv e$ wird $p \equiv e a(d e) a_{1} . e c$ $\equiv e a_{1} . e c$ (Reg. 7) $\equiv e$, weil nämlich $e a_{1} c$ nicht Null sein kann. Endlich für $x \equiv f$, wird $p \equiv f a A(a f . c d) . c f \equiv A(a f)(a f . c d) . c f($ Reg. 2) $\equiv a f . c f$ (Reg. 4) $\equiv f$ (Reg. 7). Für $x \equiv g$, $h$ oder $i$ gehe $p$ beziehlich in $g_{1}, h_{1}$ oder $i_{1}$ über, dann erhält man

$$
\begin{aligned}
& x \equiv a, c, d, e, f, g, h, i, \\
& p \equiv o, o, o, e, f, g_{1}, h_{1}, i_{1} .
\end{aligned}
$$

Ferner $q \equiv x b B$ wird zu Null für $x \equiv b$. Damit es auch für $e$ und $f$ sich vereinfache, setze man

$$
\boldsymbol{B} \equiv \boldsymbol{e f},
$$


und nehme- $b$ so an, dafs es nicht in ef falle. Dann wird für $x \equiv e, f$ der Ausdruck $q$ auch $\equiv e, f$ (Reg. 7 ); für $x \equiv g, h, i$ werde $q \equiv g_{2}, h_{2}, i_{2}$; dann erhält man

$$
\begin{aligned}
& x \equiv b, e, f, g, h, i, \\
& y \equiv o, e, f, g_{2}, h_{2}, i_{2} .
\end{aligned}
$$

Es kommt also nur noch darauf an, die Gleichung (12.) für die 5 Fälle zu befriedigen, wo

ist.

$$
\begin{aligned}
& p \equiv e, f, g_{1}, h_{1}, i_{1}, \text { und gleichzeitig } \\
& q \equiv e, f, g_{2}, h_{2}, i_{2}
\end{aligned}
$$

Man nehme noch $\boldsymbol{C} \equiv e i$ an; dann wird die genannte Gleichung für $p \equiv q \equiv e$ befriedigt, und für $p \equiv i_{1}, q \equiv i_{2}$ vereinfacht. Es ergiebt sich nämlich im ersteren Falle $p b_{1} C k q \equiv e b_{1} . e i_{1} . e k$; was 3 gerade Linien, die durch einen Punct $(e)$ gehen, als Factoren enthält, also Null ist. Ferner für $p \equiv i_{1}, q \equiv i_{2}$ erhält man $p b_{1} C k p \equiv i_{1} b_{1}\left(e i_{1}\right) k i_{2} \equiv i_{1} k i_{2}$ (Reg. 7), wenn nicht $i_{1} b_{1} e$ Null ist; im letztern Falle würde hierdurch für $k$ keine besondere Lage bedingt, im andern Falle mufs $k$ in der Geraden $i_{1} i_{2}$ liegen; dies läl'st sich daher auch im ersteren Falle annehmen.

Nun bleibt nur noch für die drei übrigen Werthpaare die Gleichung (12.) zu befriedigen; d. h. es mufs noch

$$
f b_{1} C k f=0, \quad g_{1} b_{1} C k g_{2}=0, \quad h_{1} b_{1} C k h_{2}=0
$$

sein; oder, anders geschrieben (Reg. 2,3):

$$
k f C f b_{1}=0, \quad k g_{2} C g_{1} b_{1}=0, \quad k h_{2} C h_{1} b_{1}=0 .
$$

Die erste dieser Gleichungen giebt, wenn $C f$ nicht Null ist (nach Reg. 6), $k f b_{1}=0$; und es ergiebt sich dann, dafs die geraden Linien

$$
\text { kf, } \quad \mathrm{kg}_{2} \mathrm{Cg}_{1}, \quad k h_{2} \mathrm{Ch}_{1}
$$

durch einen und denselben Punct gehen müssen, der dann gleich $b_{1}$ zu setzen ist. Sollte $\boldsymbol{C f}$ zufällig Null sein, so würde jene erstere Bedingung wegfallen. Sollen nun jene 3 gerade Linien durch einen und denselben Punct gehen, so ist dazu nöthig und ausreichend, dafs ihr Product Null sei. Also hat man zur Bestimmung von $k$ die Gleichung

$$
\text { (13.) } k f . k g_{2} C g_{1} \cdot k h_{2} C h_{1}=0 \text {. }
$$

Sie ist in Bezug auf $k$ vom dritten Grade, also ist der geometrische Ort von $k$ eine Linie dritter Ordnung. Aber diese zerfällt in 3 gerade Linien. 
Nämlich, erstens, wenn $\boldsymbol{k}$ in der geraden Linie $C$ liegt, wird $k g_{2} C \equiv k$ (Reg. 7); ebenso $k h_{2} C \equiv k$, also die linke Seite von (13.) in $k f . k g_{1} \cdot k h_{1}$; was Null ist. Ferner da $f, g_{2}, h_{2}$ in der geraden Linie $B$ liegen, so wird, wenn auch $\boldsymbol{k}$ in $\boldsymbol{B}$ liegt, $\boldsymbol{k f} \equiv \boldsymbol{k g}_{2} \equiv \boldsymbol{k} \boldsymbol{h}_{2} \equiv \boldsymbol{B}$; also geht dann die linke Seite von (13.) in $\boldsymbol{B} . \boldsymbol{B C} g_{1} . \boldsymbol{B C h}$ über, d. h. in ein Product dreier geraden Linien, die durch ein und denselben Punct $(\boldsymbol{B C})$ gehen; also ist ihr Product Null. Es wird also jeder Punct $k$, der in $B$ oder $C$ fällt, der Gleichung (13.) genügen, und mithin mufs die Linie (13.) in 3 gerade Linien zerfallen. Um die dritte zu finden, suche man zwei ihrer Puncte. Ein solcher Punct ist aus der Gleichung (13.) leicht zu finden, wenn man sie in der Form $k f\left(\operatorname{kg}_{2} C_{g_{1}}\right) h_{1} C h_{2} x=0$ schreibt (Reg. 3), und den Punct so bestimmt, dafs $\boldsymbol{k} f\left(\boldsymbol{k g} \boldsymbol{C g}_{\mathbf{1}}\right) \boldsymbol{h}_{\mathbf{1}}$ schon Null ist. Die Gleichung $\boldsymbol{k} f\left(\boldsymbol{k} g_{2} C g_{1}\right) h_{1}=0$ sagt aus, dafs die geraden Linien $k f$ und ${ } g_{2} C g_{1}$ durch den Punct $h_{1}$ gehen, d. h. dafs $k f h_{1}=0, k g C g_{1} h_{1}=0$ ist; oder diese Gleichungen umgekehrt (Reg. 3), dafs $h_{1} f k=0$ und $h_{1} g_{1} C g k=0$ ist; also liegt dann $k$ in den beiden Geraden $h_{1} f$ und $h_{1} g_{1} C g$. Der so gefundene Punct werde mit $\alpha$ bezeichnet, also

$$
\alpha \equiv h_{1} g_{1} C g\left(h_{1} f\right)
$$

gesetzt. Aus gleichem Grunde wird der Gleichung (13.) durch den Punct

$$
\beta=h_{1} g_{1} C h\left(g_{1} f\right)
$$

genügt, und da diese Puncte im Allgemeinen nicht in den Geraden $\boldsymbol{B}$ und $\boldsymbol{C}$ liegen, so ist $\alpha \beta$ die dritte der Geraden, in welche die Linie (13.) zerfällt. Liegt nun $k$ in dieser Geraden, so wird der Gleichung (13.) genügt; d. h. es gehen dann die 3 geraden Linien $k f, \mathrm{~kg}_{2} \mathrm{Cg}_{1}, \boldsymbol{k h _ { 2 }} \mathrm{Ch} \boldsymbol{h}_{1}$ durch einen und denselben Punct; derselbe heifse $b_{1}$, so wird nun die Gleichung

$$
x a A a_{1} . x b B k C b_{1} \cdot x c=0
$$

durch jeden der 9 Puncte $a \ldots i$, wenn er statt $x$ gesetzt wird, befriedigt. Denn dafs die Puncle $a, b, c, d, e, i$ ihr genügen, ist oben bewiesen; aber auch $f, y, h$ genügen ihr, denn da die geraden Linien $k f, k_{2} C g_{1}, k h_{2} C h_{1}$ durch $b_{1}$ gehen, so hat man, wenn man noch für $k f$ das ihm Gleiche $k f C f$ schreibt, die drei Gleichungen

$$
k f C f b_{1}=0, \quad k g_{2} C g_{1} b_{1}=0, \quad k h_{2} C h_{1} b_{1}=0,
$$

oder umgeordnet (Reg. 2, 3) :

$$
f b_{1} C k f=0, \quad g_{1} b_{1} C k g_{2}=0, \quad h_{1} b_{1} C k h_{2}=0 .
$$


Aber $f, g_{1}, h_{1}$ waren die Werthe von $p$, und $f, g_{2}, h_{2}$ die von $q$, wenn $x$ beziehlich die Werthe $f, g, h$ annahm, also wird die Gleichung (12.) auch für $x \equiv f, g, h$ erfüllt, mithin auch die mit (12.) identische Gleichung (11); und die Aufgabe ist gelöst. Da übrigens $k$ in $\alpha \beta$ und in $i_{1} i_{2}$ lag, so hat man $k \equiv \alpha \beta\left(i_{1} i_{2}\right)$, und da $b_{1}$ in $k f$ und in $k g_{2} C g_{1} \operatorname{lag}$, so hat man $b_{1} \equiv k g_{2} C g_{1}(k f)$. Somit hat sich folgender Satz ergeben:

„Wenn $a, \ldots i$ neun beliebige Puncte sind, und

$$
A \equiv d e, a_{1} \equiv a f . c d, B \equiv e f ;, C \equiv e i_{1}, k \equiv \alpha \beta\left(i_{1} i_{2}\right), b_{1} \equiv k g_{2} C g_{1}(k f)
$$

gesetzt wird, wo $g_{1}, h_{1}, i_{1}$ die Puncte sind, in welche sich $x a A a_{1} . x c$ verwandelt, wenn man statt $x$ nach und nach die Puncte $y, h, i$ substituirt, und $g_{2}, i_{2}$ die Puncte, in welche sich $x \boldsymbol{b B}$ verwandelt, wenn man statt $x$ beziehlich die Puncte $g$ und $i$ setzt, und wo

$$
\alpha \equiv h_{1} g_{1} C g\left(h_{1} f\right) ; \quad \beta \equiv h_{1} g_{1} C h\left(g_{1} f\right)
$$

ist: so ist

$$
x a A a_{1} . x b B k C b_{1}, x c=0
$$

die Gleichung der Curve dritter Ordnung, welche durch die gegebenen 9 Puncte $a$... geht."

Es läfst sich die gefundene Gleichung nach (\$. 6.) nun auch auf die einfachere Form (2.) zurückführen; was ich jedoch nicht weiter verfolge.

Es drückt die oben gefundene Gleichung zugleich die lineale Beziehung aus, welche zwischen 10 Puncten $a, b, \ldots i, x$ herrschen mufs, damit sie in einer Curve dritter Ordnung liegen; also die lineale Eigenschaft des einer solchen eingeschriebenen Zelinecks.

Es ist dies die einfachste Eigenschaft dieses Zehnecks, die ich bisher bemerkt habe, obgleich die Gleichung (11.), welche dieselbe darstellt, nachdem man statt der darin vorkommenden Gröfsen die angegebenen Ausdrücke substituirt hat, bis nur noch die 10 Ecken des Zehnecks darin vorkommen, noch immer 351 Factoren enthält, indem nämlich $a, c, d$ je $62 \mathrm{mal}, x, b, e, f, g$, $h, i$ beziehlich $3,5,44,43,24,21,25$ mal darin vorkommen.

\section{\$. 9.}

Fernere Sätze über das Zehneck in einer Curve dritter Ordnung.

Ich theile zum Schlusse noch einige hierhergehörige Sätze mit, ohne den leicht sich ergebenden Beweis beizufügen.

Crelle's Journal f. d. M. Bd. LII. Heft 3. 
I. Zwischen 10 Puncten $a \ldots k$ einer Curve dritter Ordnung bestehen folgende Eigenschaften:

a) Wenn man durch 4 der Puncte (z. B. a, b, c, d) 6 Kegelschnitte legl, welche aufserdem beziehlich durch je einen der übrigen 6 Puncte $(e \ldots k)$ gehen, so läfst sich stets ein eilfter Punct $(l)$ von der Art finden, dafs jene 6 Kegelschnitte mit den 6 Strahlen, die von dem eilften Puncte $(b)$ nach diesen 6 Puncten $(e \ldots k)$ gehen, projectivisch sind.

b) Wenn man zwei von den Puncten (z. B. $\boldsymbol{a}, \boldsymbol{b}$ ) zu Mittelpuncten zweier Strahlbüschel von je 8 Strahlen macht, deren entsprechende Strahlen sich in den übrigen 8 Puncten $(c \ldots k)$ schneiden, so lassen sich stets zwei Gerade $\boldsymbol{A}$ und $\boldsymbol{B}$ finden, welche jenen Strahlbüscheln projectivisch sind, und welche die Beschaffenheit haben, dafs jede Verbindungslinie zweier entsprechender Puncte dieser Geraden durch den Punct geht, in welchem sich die diesen Puncten entsprechenden Strahlen jener Strahlbüschel schneiden.

c) Wenn man drei von den Puncten (z. B. $a, b, c)$ zu Mittelpuncten dreier Strahlbüschel von je 7 Strahlen macht, deren entsprechende Strahlen sich in den 7 übrigen Puncten $(d \ldots k)$ treffen, so lassen sich allemal 3 siebenpunctige Gerade finden, die beziehlich den 3 Strahlenbüscheln projectivisch sind, und von deren Puncten je 3 entsprechende in gerader Linie liegen.

d) Wenn man, wie in $c$ ), drei der Puncte zu Mittelpuncten dreier Strahlenbüschel von je 7 Strahlen macht, deren entsprechende Strahlen sich in den 7 übrigen Puncten treffen, so lassen sich stets 3 andere Strahlbüschel von je 7 Strahlen finden, die den ersteren projectivisch sind, und von deren Strahlen je 3 entsprechende durch einen und denselben Punct gehen.

e) Legt man durch die 10 Puncte zwei gesonderte, (d.h. nicht ganz oder theilweise zusammenfallende) Linien vierter Ordnung, so schneiden sich diese aufserdem in 6 Puncten, durch welche sich ein Kegeischnitt legen läfst.

II. Umgekehrt: Wenn 10 Puncte eine der genannten 5 Eigenschaften besitzen, so liegen sie in einer Linie dritter Ordnung.

Von diesen 5 Eigenschaften habe ich die erste schon früher (Bd. 42. S. 208) in entsprechender Weise für Curven beliebiger Ordnung nachgewiesen, und namentlich auch auf Curven vierter Ordnung angewandt (Bd.44. S. $21 \mathrm{ff}$.). Die folgenden 3 Eigenschaften gehen aus den 3 Hauptformen der linealen Gleichungen dritten Grades, wie sie an die Spitze dieses Aufsatzes gestellt sind, 
hervor. Die fünfte Eigenschaft, welche sich am leichtesten durch Functionsverknüpfungen (vergl. Bd. 42. S. $204 \mathrm{ff}$.) ableiten läfst, erhält ein besonderes Interesse, wenn man als die betreffenden Curven vierter Ordnung 2 Vereine von je 2 Kegelschnitten annimmt, von denen jeder Kegelschnitt durch 5 der gegebenen Puncte geht, der demselben Vereine angehörige also durch die 5 übrigen Puncte. Es zeigt sich diese Eigenschaft der des Seclesecks, welches einem Kegelschnitte eingeschrieben ist, ganz entsprechend, indem sich der Pascalsche Satz zu folgendem Satze erweitern läfst.

„Wenn man durch 6 Puncte eines Kegelschnitts 2 gesonderte Linien dritter Ordnung legt, so treffen sich diese aufserdem in 3 Puncten, die in gerader Linie liegen. Und nimmt man hierbei als die betreffenden Curven 2 Vereine von je 3 Geraden an, so hat man den Pascalschen Satz in der gewöhnlichen Form."

Stettin, den 15. April 1855. 